\title{
A Comparative Preliminary Study on CT Contrast Attenuation Gradient Versus Invasive FFR in Patients with Unstable Angina
}

\author{
Marius Orzan ${ }^{1}$, Mihaela Dobra $^{1,2}$, Monica Chițu$^{1}$ \\ ${ }^{1}$ Laboratory of Advanced Research in Multimodal Cardiac Imaging, University of Medicine and Pharmacy, Tîrgu Mureș, Romania \\ ${ }^{2}$ Department of Computational Imaging, Cardio Med Medical Center, Tîrgu Mureș, Romania
}

\section{ABSTRACT}

The aim of this preliminary study was to assess the effectiveness of transluminal contrast attenuation gradient (TAG) determined by computed tomographic angiography (CTA), for the evaluation of the functional significance of coronary artery stenoses in patients with acute coronary syndromes produced by vulnerable coronary plaques, and to demonstrate the correlation between this new parameter and the vulnerability markers of the culprit lesions. Material and methods: This is a preliminary pilot study on 10 patients with acute coronary syndromes - unstable angina type, who underwent CTA for the assessment of coronary lesions, followed by invasive angiography and the determination of fractional flow reserve (FFR) prior to a revascularization procedure. Patients were divided into 2 groups, according to their FFR values: Group 1 consisted of 6 patients with an FFR value $<0.8$ (functionally significant lesion), and Group 2 consisted of 4 patients who presented an FFR value $>0.8$ (functionally non-significant lesion). Results: FFR values were $0.64 \pm 0.07,95 \%$ CI: $0.5-0.7$ in Group 1, and $0.86 \pm 0.05,95 \% \mathrm{CI}: 0.7-0.9$ in Group 2. Plaques associated with an FFR $<0.8$ presented a higher amount of plaque volume $(192.7 \pm 199.7 \mathrm{~mm} 3 \mathrm{vs} .42 .1 \pm 27.3 \mathrm{~mm} 3, \mathrm{p}=0.1)$, necrotic core $(66.7 \pm 72.9 \mathrm{~mm} 3$ vs. $10.0 \pm 9.3 \mathrm{~mm} 3, \mathrm{p}=0.1)$, and fibro-fatty tissue $(29.7 \pm 37.4 \mathrm{~mm} 3 \mathrm{vs}$. $6.2 \pm 3.8 \mathrm{~mm} 3, \mathrm{p}=0.2$ ). At the same time, TAG significantly correlated with the presence of a functionally significant lesion. Coronary lesions associated with low FFR presented significantly higher values of TAG along the plaque as compared with lesions with FFR values $>0.8$ (TAG values $22.1 \pm 5.8 \mathrm{HU}$ vs. $11.7 \pm 2.5 \mathrm{HU}, \mathrm{p}=0.01$ ). Linear regression identified a significant correlation between TAG and FFR values as a measure of functional significance of the lesion $(r=0.7, p=0.01)$. Conclusions: Contrast attenuation gradient along the culprit lesion, determined by CTA, correlates with the FFR values and with CT markers of plaque vulnerability, indicating that the presence of vulnerability features inside a coronary plaque could increase the functional significance of a coronary lesion.

Keywords: contrast atenuation, vulnerable coronary plaque, computed tomographic angiography, fractional flow reserve

\section{ARTICLE HISTORY}

Received: April 7, 2017

Accepted: May 19, 2017

\section{CORRESPONDENCE}

Mihaela Rațiu

Str. 22 Decembrie 1989 nr. 76

540124 Tîrgu Mureș, Romania

Tel: +40 265217333

E-mail: d_a_mihaela@yahoo.com 


\section{INTRODUCTION}

Coronary atherosclerosis represents one of the most devastating diseases worldwide, and acute coronary syndromes (ACS) are the most severe form of this condition. The costs related to the complex investigations of ACS and the associated treatment are constantly increasing; however, the mortality of patients with acute myocardial infarction remains very high, reaching $20 \%$ in the absence of timely initiation of reperfusion treatment., ${ }^{1,2}$

New cardiac imaging technologies have been developed in recent years in an attempt to identify imaging markers associated with an increased vulnerability of the coronary plaques, hoping that prompt treatment of these lesions could prevent the progression of the disease towards the development of an acute coronary event. Such a technique is represented by computed tomography angiography (CTA), which is able not only to identify the presence of vulnerability markers inside the plaque, but also to quantify the amount of its components on the basis of CT density. However, the decision to stent or not a vulnerable plaque that is not hemodynamically significant remains challenging for the interventionist. ${ }^{3-5}$

Usually, the severity of a coronary artery stenosis is estimated on the basis of coronary angiography, by calculating the narrowing degree of the coronary lumen. However, intravascular ultrasound (IVUS) studies demonstrated that not all culprit lesions that have triggered an acute coronary syndrome produce a significant narrowing of the coronary lumen. ${ }^{6,7}$ At the same time, the PROSPECT trial demonstrated that the amount of plaque burden evaluated by IVUS was the strongest predictor of mortality in patients with vulnerable plaques. ${ }^{8}$ Also, the COURAGE trial demonstrated the non-superiority of visual cineangiography-guided revascularizations compared to the correct medication in regards to mortality, myocardial infarction, and other cardiovascular complications. ${ }^{9,10}$

At the same time, a new technology represented by the estimation of fractional flow reserve (FFR) has been suggested and implemented in cath labs in recent years, in order to provide a robust tool for the assessment of the functional significance of a coronary stenosis. FFR, an invasive technique that measures the intraluminal pressure after stenosis compared to the pressure proximal to stenosis with the aid of a special transducer similar to a coronary guide, rapidly became a gold standard for assessing the functional significance of a coronary artery stenosis. FFR is defined as the ratio between the mean distal and proximal pressure to stenosis, and a rate of less than 0.8 is considered a strong indicator of a significant steno- sis. ${ }^{11,12}$ The FAME and FAME II trials demonstrated that a percutaneous coronary intervention (PCI) strategy based on the functional signification of the coronary stenosis is preferable to the classical strategy based on angiographic aspect.11,13 However, the exact relation between the functional significance of a coronary lesion and the vulnerability degree of this plaque is still under investigation.

Multiple CTA-derived markers have been proposed for plaque characterization, in stable or unstable plaques. ${ }^{14,15}$ The plaque burden, the amount of low-density plaque, or the remodeling index are vulnerability markers easily calculated on CTA images using modern post-processing software. At the same time, it has been proposed that the degree of attenuation of contrast material along the stenosis, determined by CTA, could serve as a functional parameter expressing the hemodynamic significance of a coronary artery stenosis. ${ }^{12,16,17}$

The aim of this preliminary study was to assess the effectiveness of transluminal contrast attenuation gradient (TAG) determined by CTA, for the evaluation of the functional significance of coronary artery stenoses in patients with acute coronary syndromes produced by vulnerable coronary plaques, and to demonstrate the correlation between this new parameter and the vulnerability markers of the culprit lesions.

\section{MATERIAL AND METHODS}

This is a preliminary pilot study on 10 patients with ACS unstable angina type, who underwent CTA for the assessment of coronary lesions, followed by invasive angiography and determination of FFR prior to a PCI procedure.

Patients with other forms of ACS (acute myocardial infarction, with or without ST-segment elevation), hemodynamically unstable patients, or patients with significant kidney dysfunction were excluded from the study.

The study protocol was approved by the ethics committee of the institution where the procedures were performed, and written informed consent was obtained from each participant included in the study. All study procedures have been carried out according to the principles stipulated in the Declaration of Helsinki.

Prior to the CTA examination, all patients received betablockers, in order to obtain a heart rate below $60 / \mathrm{min}$, and $1 \mathrm{~mL} / \mathrm{kg}$ of ionic contrast media was administrated through an intravenous 18-gauge line at a flow rate of $6.1 \mathrm{~mL} / \mathrm{s}$, followed by a $150 \mathrm{~mL}$ saline chaser at the same flow rate.

Image acquisition was performed with a Siemens Somatom 128-slice CT equipment (Siemens, Erlangen, Germany), followed by automated and manual reconstruction 
and evaluation of the coronary artery. Post-processing and image analysis for the quantification of coronary plaque components have been carried out using the QANgioCT RE software (Medis, Leiden, The Netherlands), which allowed atherosclerotic plaque characterization and quantification of plaque components such as: plaque volume, lesion length, mean and maximum plaque burden, fibrous fatty volume, necrotic core volume, \% stenosis, and TAG expressed in mean $\mathrm{HU} / \mathrm{mm}$.

TAG was calculated as the difference in the contrast density along the coronary stenosis, between the segment located $1 \mathrm{~mm}$ proximal to the stenosis and the segment located $1 \mathrm{~mm}$ distal to the lesion (Figure 1 ).

Following CTA examination, all patients underwent invasive angiography and FFR calculation using the Optis Integrated System (St Jude Medical, St Paul, Minnesota, USA), and Aeris Agile Tip pressure wire catheters (St Jude Medical, St Paul, Minnesota, USA) for invasive FFR determination.

Patients were divided into 2 groups, according to their FFR values: Group 1 consisted of 6 patients with an FFR value $<0.8$ (functionally significant lesion) and Group 2 consisted of 4 patients who presented an FFR value $>0.8$ (functionally non-significant lesion).

Statistical analysis was performed using the GraphPad InStat 3.0 software (GraphPad Software, San Diego, USA). For comparing the characteristics of all patients between Group 1 and Group 2, Fisher's exact test (or Student's ttest for age) was used. Continuous values were expressed as mean \pm standard deviation, and statistical significance was determined using the Mann-Whitney test. Linear regression was used for assessing the correlation between FFR values and TAG. Statistical significance was considered for $\mathrm{p}$ values $<0.05$ and all $\mathrm{p}$ values were two-sided.

\section{RESULTS}

Baseline characteristics of the study population are presented in Table 1. There were no statistically significant differences between the study groups in relation to age, gender, and cardiovascular risk factors (smoking status, hypertension, obesity, dyslipidemia) (Table 1).
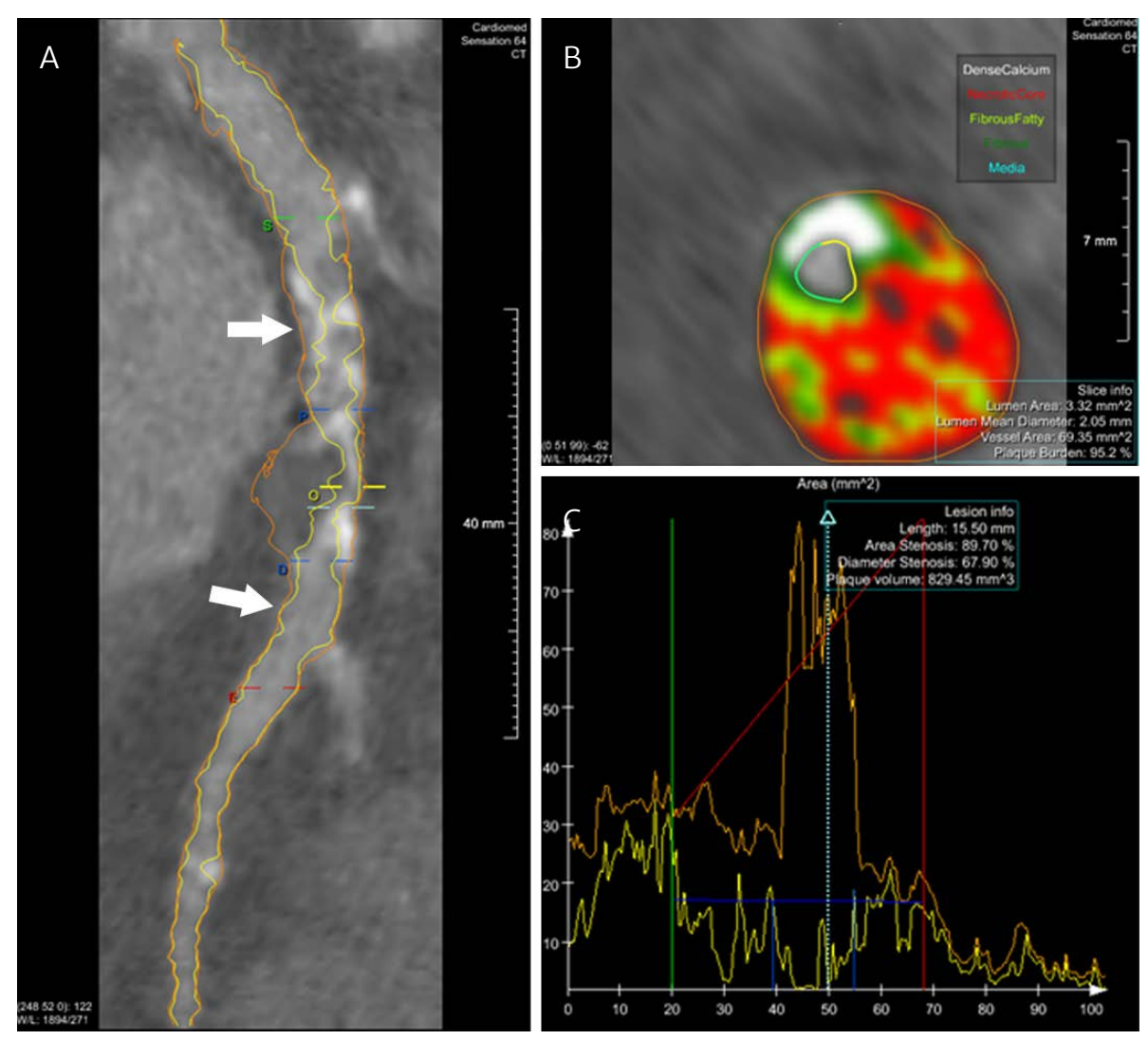

FIGURE 1. Automated post-processing of a coronary vulnerable plaque. A - reference points for calculation of the attenuation gradient between the segment located proximal and distal to the plaque (arrows); B - color coded representation of plaque components based on contrast density and quantification of plaque burden, fibrofatty tissue and necrotic core; $\mathbf{C}$ graphical representation of quantification of plaque components based on contrast density 
TABLE 1. Patient characteristics in the study groups

\begin{tabular}{lccc}
\hline & $\begin{array}{c}\text { Group 1 } \\
\text { FFR }<\mathbf{0 . 8} \\
\mathbf{n = 6}\end{array}$ & $\begin{array}{c}\text { Group 2 } \\
\text { FFR }>\mathbf{0 . 8} \\
\mathbf{n}=\mathbf{4}\end{array}$ & p value \\
\hline Age, years & $68.5 \pm 7.2$ & $69.2 \pm 9.5$ & 0.7 \\
Gender, male & $3(50.0 \%)$ & $3(75.0 \%)$ & 0.5 \\
Hypertension & $3(50.0 \%)$ & $2(50.0 \%)$ & 1 \\
Hyperlipidemia & $3(50.0 \%)$ & $2(50.0 \%)$ & 1 \\
Obesity (BMI $\left.>25 \mathrm{~km} / \mathrm{m}^{2}\right)$ & $2(33.3 \%)$ & $2(50.0 \%)$ & 1 \\
Smoker* & $3(50.0 \%)$ & $1(25.0 \%)$ & 0.5 \\
Diabetes & $1(16.5 \%)$ & $1(25.0 \%)$ & 1 \\
\hline
\end{tabular}

*past or present

FFR values were $0.64 \pm 0.07,95 \%$ CI: $0.5-0.7$ in Group 1 , and $0.86 \pm 0.05,95 \%$ CI: $0.7-0.9$ in Group 2.

The CT analysis of plaque characteristics revealed that lesion length and stenosis severity were statistically significantly higher in Group 1 as compared with Group 2 ( $\mathrm{p}<0.0001)$. At the same time, functionally significant plaques presented an elevated expression of vulnerabil- ity markers inside the plaques as compared to functionally non-significant plaques (Figure 2). Plaques associated with an $\mathrm{FFR}<0.8$ presented a larger plaque volume $\left(192.7 \pm 199.7 \mathrm{~mm}^{3}\right.$ vs. $\left.42.1 \pm 27.3 \mathrm{~mm}^{3}, \mathrm{p}=0.1\right)$, necrotic core $\left(66.7 \pm 72.9 \mathrm{~mm}^{3}\right.$ vs. $\left.10.0 \pm 9.3 \mathrm{~mm}^{3}, \mathrm{p}=0.1\right)$ and fibro-fatty tissue $\left(29.7 \pm 37.4 \mathrm{~mm}^{3}\right.$ vs. $6.2 \pm 3.8 \mathrm{~mm}^{3}$, $\mathrm{p}=0.2$ ) (Table 2). However, the differences were not sta-
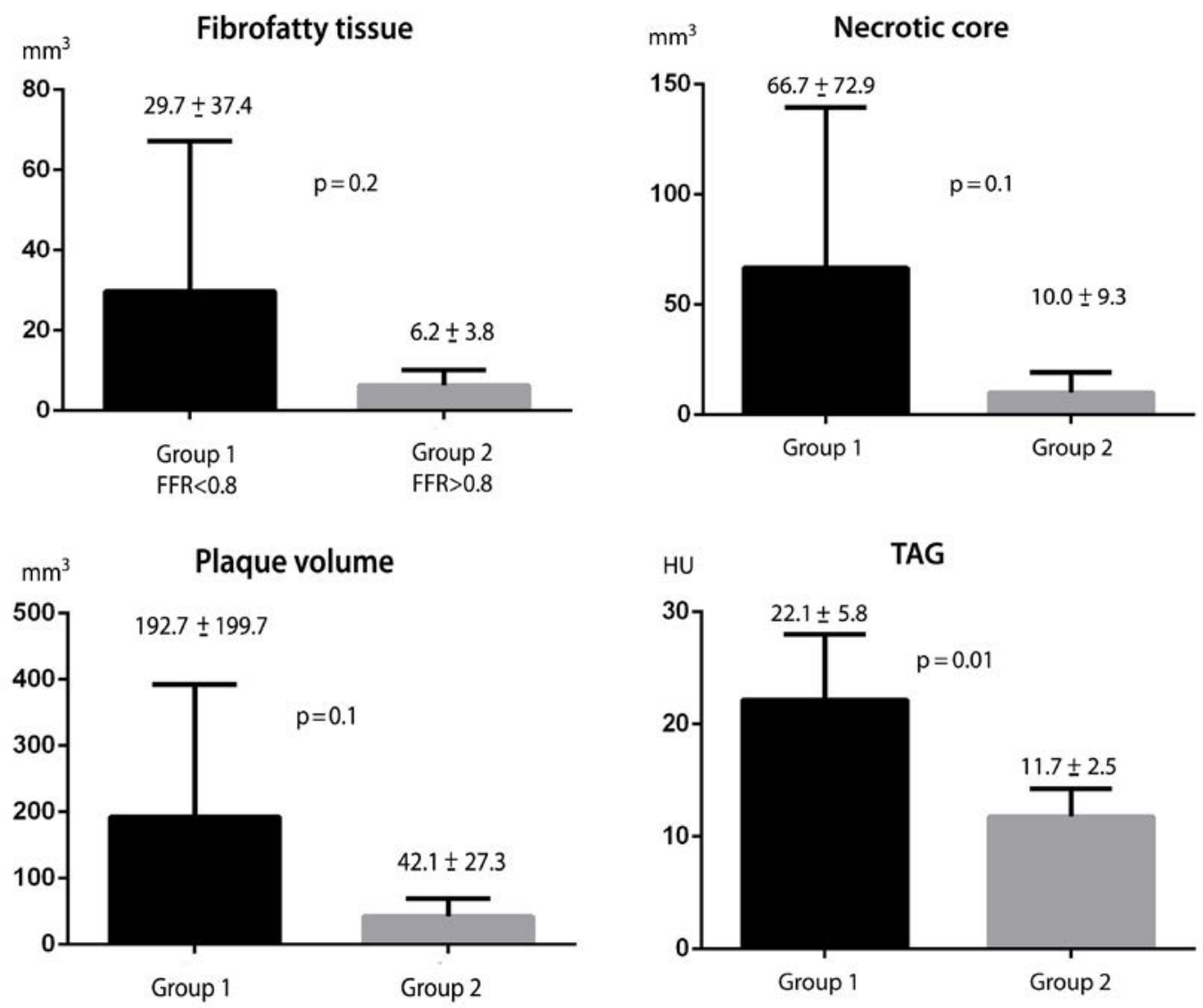

FIGURE 2. Plaque characteristics and TAG values in the culprit lesions of the group with FFR $<0.8$ vs. the group with FFR $>0.8$ 
TABLE 2. Plaque characteristics in the study groups

\begin{tabular}{|c|c|c|c|}
\hline & $\begin{array}{c}\text { Group } 1 \\
\text { FFR }<0.8 \\
\text { n }=6\end{array}$ & $\begin{array}{c}\text { Group } 2 \\
\text { FFR }>0.8 \\
n=4\end{array}$ & $\mathrm{p}$ value \\
\hline Lesion length (mm) & & & $<0.0001$ \\
\hline Mean \pm SD & $9.9 \pm 3.6$ & $3.2 \pm 1.7$ & \\
\hline $95 \%$ confidence interval & $6.1-13.7$ & $0.4-6.0$ & \\
\hline \% stenosis (\%) & & & $<0.0001$ \\
\hline Mean \pm SD & $82.7 \pm 8.3$ & $40.1 \pm 5.1$ & \\
\hline $95 \%$ confidence interval & $74.0-91.5$ & $32.0-48.3$ & \\
\hline Plaque burden (\%) & & & 0.4 \\
\hline Mean \pm SD & $76.7 \pm 8.2$ & $72.3 \pm 6.6$ & \\
\hline 95\% confidence interval & $68.1-85.3$ & $61.7-82.9$ & \\
\hline Plaque volume $\left(\mathrm{mm}^{3}\right)$ & & & 0.1 \\
\hline Mean \pm SD & $192.7 \pm 199.7$ & $42.1 \pm 27.3$ & \\
\hline 95\% confidence interval & $-16.8-402.3$ & $-1.3-85.5$ & \\
\hline Plaque thickness ( $\mu \mathrm{m})$ & & & 0.6 \\
\hline Mean \pm SD & $3.3 \pm 1.6$ & $2.9 \pm 1.1$ & \\
\hline 95\% confidence interval & $1.5-5.1$ & $1.2-4.7$ & \\
\hline TAG (HU) & & & 0.01 \\
\hline Mean \pm SD & $22.1 \pm 5.8$ & $11.7 \pm 2.5$ & \\
\hline 95\% confidence interval & $16.0-28.2$ & $7.7-15.7$ & \\
\hline Fibro-fatty tissue $\left(\mathrm{mm}^{3}\right)$ & & & 0.2 \\
\hline Mean \pm SD & $29.7 \pm 37.4$ & $6.2 \pm 3.8$ & \\
\hline $95 \%$ confidence interval & $-9.5-69.0$ & $0.1-12.4$ & \\
\hline Necrotic core tissue $\left(\mathrm{mm}^{3}\right)$ & & & 0.1 \\
\hline Mean \pm SD & $66.7 \pm 72.9$ & $10.0 \pm 9.3$ & \\
\hline $95 \%$ confidence interval & $-9.8-143.2$ & $-4.7-24.8$ & \\
\hline
\end{tabular}

TAG - trans-stenotic attenuation gradient

tistically significant, probably due to the limited number of cases.

At the same time, TAG significantly correlated with the presence of a functionally significant lesion. Coronary lesions associated with low FFR presented significantly higher values of TAG along the plaque as compared with lesions with FFR values >0.8 (TAG values $22.1 \pm 5.8 \mathrm{HU}$ vs. $11.7 \pm 2.5 \mathrm{HU}, \mathrm{p}=0.01$ ) (Figure 3).

Linear regression identified a significant correlation between TAG and FFR values as a measure of functional significance of the lesion $(r=0.7, p=0.01)$, indicating that the decrease in FFR in functionally significant plaques is directly correlated with the decrease in contrast density along the plaque, as expressed by an increased contrast gradient along the lesion.

\section{DISCUSSION}

CTA has become one of the most extensively used and useful imaging techniques in cardiology, and its relevance in patients with vulnerable coronary plaques is nowadays widely accepted..$^{18}$

Despite of a multitude of studies that assessed the role of CTA in evaluating plaque vulnerability, according to our knowledge there are no published data so far regarding the role of CTA in assessing vulnerability markers in relation to the functional significance of a coronary lesion. ${ }^{19-23}$

FFR remains the gold standard for assessing the hemodynamic significance of a coronary artery stenosis; however, this remains an invasive technique that could prove to be unnecessary in cases with no significant lesions. Therefore the validation of new imaging-derived markers able to assess the functional significance of a coronary lesion using a noninvasive route could play a significant role in the reduction of unnecessary invasive coronary interventions.

The CT-based calculation of FFR (CT-FFR) has been also introduced in recent years as a new parameter for the estimation of lesion severity on a noninvasive route, using computational fluid dynamics and the simulation of 


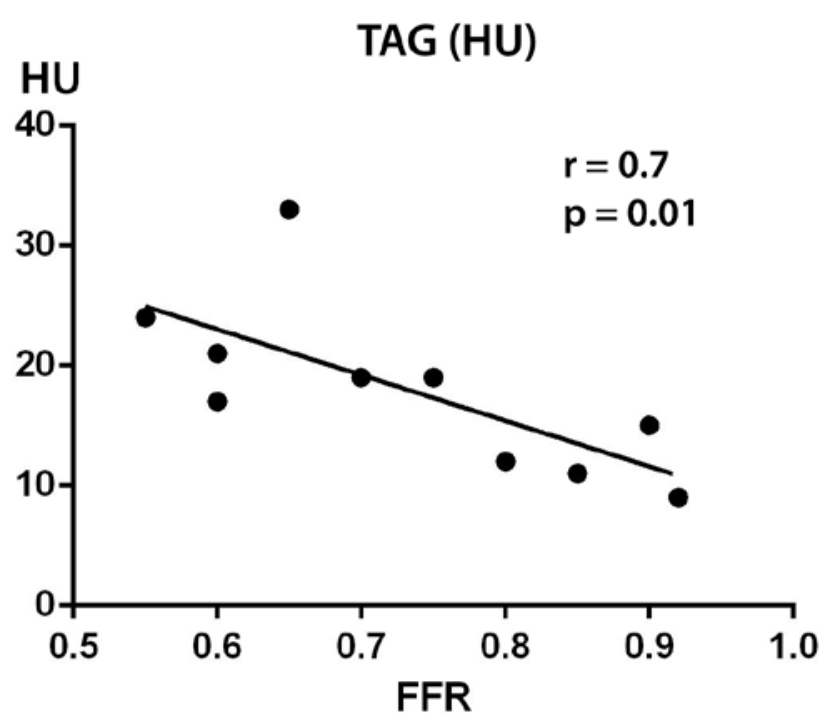

FIGURE 3. Correlation between TAG and FFR in culprit lesions of patients with unstable angina

coronary flow. ${ }^{24}$ Also, coronary shear stress, calculated using computational fluid dynamics, has been proposed to be associated with plaque vulnerability in unstable coronary lesions. Several recent studies proved that unstable plaques exhibit reduced shear stress in the region located immediately distal to the vulnerable coronary plaque. ${ }^{25}$ However, all these techniques based on coronary flow simulation and computational post-processing are timeconsuming and require significant skills and experienced operators, being difficult to be performed in a timely fashion, as requested in many cardiovascular emergencies.

In recent studies, TAG and CT-FFR have shown their superiority as compared with invasive FFR in intermediate lesions with a stenosis degree between $30 \%$ and $70 \%$, showing good sensitivity and specificity for discriminating between significant and non-significant lesions. ${ }^{12}$

As compared with the gold standard (FFR), TAG presents the advantage that it does not require the administration of vasodilators drugs, while FFR requires adenosine administration. It is also important to mention that the presence of coronary calcification could impact the quality of TAG measurement, an impediment that is not encountered when using the invasive FFR approach. Corrective applications of CT post-processing techniques have been proposed to overcome this disadvantage, such as TAG with corrected contrast opacification (TAG-CCO) and TAG with the exclusion of calcified coronary segments (TAG-ExC). Some studies have shown that in comparison to classical coronary angiography, TAG-ExC can improve the coronary CT angiography specificity, while TAG-CCO has a limited contribution in addition of the information of CTA alone. ${ }^{23,26}$

In this pilot study, we demonstrated that TAG could be considered a new imaging-derived parameter able to estimate the functional significance of coronary lesions, being significantly increased in coronary lesions classified as significant based on FFR assessment, the currently validated gold standard. At the same time, TAG was associated with CT markers of plaque vulnerability, such as necrotic core or plaque volume, indicating that TAG can be considered a marker indirectly associated with plaque vulnerability. This could also open the hypothesis that vulnerable coronary plaques have a higher impact on the functional significance of a coronary lesion, probably via complex mechanisms related to the alteration of the local dynamic forces in the coronary flow, a process in which local inflammation could play a certain role.

\section{LIMITATION OF THE STUDY}

One major limitation of this study is that the TAG and FFR evaluations were made in different moments; therefore, the information provided by the two imaging tools could not be entirely superposable.

On the other hand, the small number of patients included in this pilot study makes it hard to generalize the conclusions. However, this study opens new perspectives for challenging a hypothesis that could be further explored in larger scale studies.

\section{CONCLUSION}

In patients with unstable angina, contrast attenuation gradient along the culprit lesion, determined by CTA, could serve as a promising tool for the estimation of the functional significance of the lesion, being highly correlated with FFR values. At the same time, TAG correlates with CT markers of plaque vulnerability, indicating that the presence of vulnerability features inside a coronary plaque could increase the functional significance of a coronary lesion.

\section{CONFLICT OF INTEREST}

Nothing to declare.

\section{REFERENCES}

1. Barton GR, Irvine L, Flather M, et al. Economic Evaluation of Complete Revascularization for Patients with Multivessel 
Disease Undergoing Primary Percutaneous Coronary Intervention.Value Health. 2017;20:745-751. doi: 10.101622.

2. Dubey G, Verma SK, Bahl VK. Primary percutaneous coronary intervention for acute ST elevation myocardial infarction: Outcomes and determinants of outcomes: A tertiary care center study from North India. Indian Heart J. 2017;69:294298. doi: 10.1016/j.ihj.2016.11.322.

3. Tonino PA, Fearon WF, De Bruyne B, et al. Angiographic versus functional severity of coronary artery stenoses in the FAME study fractional flow reserve versus angiography in multivessel evaluation. J Am Coll Cardiol. 2010;55:2816-2821. doi: 10.1016/j.jacc.2009.11.096.

4. De Bruyne B, Pijls NH, Kalesan B, et al. Fractional flow reserve-guided PCI versus medical therapy in stable coronary disease. N Engl J Med. 2012;367:991-1001. doi: 10.1056/ NEJMoa1205361.

5. Boden WE, O'Rourke RA, Teo KK, et al. Optimal medical therapy with or without PCI for stable coronary disease. N Engl J Med. 2007;356:1503-1516. doi: 10.1056/NEJMoa070829.

6. Kwon JE, Lee WS, Mintz GS, et al. Multimodality Intravascular Imaging Assessment of Plaque Erosion versus Plaque Rupture in Patients with Acute Coronary Syndrome. Korean Circ J. 2016;46:499-506. doi: 10.4070/kcj.2016.46.4.499.

7. Dong L, Mintz GS, Witzenbichler B, et al. Comparison of plaque characteristics in narrowings with ST-elevation myocardial infarction (STEMI), non-STEMI/unstable angina pectoris and stable coronary artery disease (from the DAPTDES IVUS Substudy). Am J Cardiol. 2015;115:860-866. doi: 10.1016/j.amjcard.2015.01.008.

8. Zheng B, Mintz GS, McPherson JA, et al. Predictors of Plaque Rupture Within Nonculprit Fibroatheromas in Patients With Acute Coronary Syndromes: The PROSPECT Study. JACC Cardiovasc Imaging. 2015;8:1180-1187. doi: 10.1016/j. jcmg.2015.06.014.

9. Acharjee S, Teo KK, Jacobs AK et al. Optimal medical therapy with or without percutaneous coronary intervention in women with stable coronary disease: A pre-specified subset analysis of the Clinical Outcomes Utilizing Revascularization and Aggressive druG Evaluation (COURAGE) trial. Am Heart J. 2016;173:108-117. doi: 10.1016/j. ahj.2015.07.020.

10. Boden WE, O'Rourke RA, Teo KK, et al. Optimal medical therapy with or without PCI for stable coronary disease. N Engl J Med. 2007;356:1503-1516. doi: 10.1056/NEJMoa070829.

11. Andreini D, Pontone G, Mushtaq S, et al. A long-term prognostic value of coronary CT angiography in suspected coronary artery disease. JACC Cardiovasc Imaging. 2012;5:690701. doi: 10.1016/j.jcmg.2012.03.009

12. Cademartiri F, Seitun S, Clemente A, et al. Myocardial blood flow quantification for evaluation of coronary artery disease by computed tomography. Cardiovasc Diagn Ther. 2017;7:129150. doi: 10.21037/cdt.2017.03.22.

13. De Bruyne B, Pijls NH, Kalesan B, et al. Fractional flow reserveguided PCI versus medical therapy in stable coronary disease. N Engl J Med. 2012;367:991-1001. doi: 10.1056/NEJMoa1205361

14. Mollet N, Maffei E, Martini C, et al. Coronary plaque burden in patients with stable and unstable coronary artery disease using multislice CT coronary angiography. Radiol Med. 2011;116:1174-1187. doi: 10.1007/s11547-011-0722-5.

15. Dalager MG, Bøttcher M, Thygesen J, Andersen G, Bøtker HE. Different Plaque Composition and Progression in Patients with Stable and Unstable Coronary Syndromes Evaluated by Cardiac CT. Biomed Res Int. 2015;2015:401357. doi: 10.1155/2015/401357.

16. Peng K. Transluminal attenuation gradient and corrected models in coronary CT angiography for determining stenosis severity: a primary study using dual-source CT. Clin Radiol. 2017;72:508-516. doi: 10.1016/j.crad.2017.01.003.

17. Kim HJ, Kim SM, Choi JH, et al. Influence of scan technique on intracoronary transluminal attenuation gradient in coronary CT angiography using 128-slice dual source CT: multi-beat versus one-beat scan. Int J Cardiovasc Imaging. 2017;33:937946. doi: 10.1007/s10554-017-1078-2.

18. De Cecco CN, Caruso D, Baumann S, et al. Coronary CT angiography derived morphological and functional quantitative plaque markers correlated with invasive fractional flow reserve for detecting hemodynamically significant stenosis. J Cardiovasc Comput Tomogr. 2016;10:199-206. doi: 0.1016/j.jcct.2016.03.002.

19. Choi JH, Min JK, Labounty TM, et al. Intracoronary transluminal attenuation gradient in coronary CT angiography for determining coronary artery stenosis. JACC Cardiovasc Imaging. 2011;4:1149-57. doi: 10.1016/j.jcmg.2011.09.006.

20. Benedek T, Jako B, Benedek I. Plaque Quantification by Coronary CT and Intravascular Ultrasound Identifies a Low CT Density Core as a Marker of Plaque Instability in Acute Coronary Syndromes. Int Heart J. 2014;55:22-29.

21. Benedek I, Bucur O, Benedek T. Intracoronary infusion of mononuclear bone marrow derived stem cells is associated with lower plaque burden after 4 years. J Atheroscler Thromb. 2014;21:217-229.

22. Benedek T, Gyöngyösi M, Benedek I. Multislice computed tomographic coronary angiography for quantitative assessment of culprit lesions in acute coronary syndromes. Can J Cardiol. 2013;29:364-371. doi: 10.1016/j.cjca.2012.11.004.

23. Chow BJ, Kass M, Gagné O, et al. Can differences in corrected coronary opacification measured with computed tomography predict resting coronary artery flow? J Am Coll Cardiol. 2011;57:1280-1288. doi: 10.1016/j.jacc.2010.09.072

24. Collet C, Onuma Y, Serruys PW, et al. Integration of noninvasive functional assessments with anatomical risk stratification in complex coronary artery disease: the noninvasive functional SYNTAX score. Cardiovasc Diagn Ther. 2017;7:151-158. doi: 10.21037/cdt.2017.03.19.

25. Han D, Starikov A, Ó Hartaigh B, et al. Relationship Between Endothelial Wall Shear Stress and High-Risk Atherosclerotic Plaque Characteristics for Identification of Coronary Lesions That Cause Ischemia: A Direct Comparison With Fractional Flow Reserve. J Am Heart Assoc. 2016;5pii:e004186.

26. Wong DT, Ko BS, Cameron JD, et al. Transluminal attenuation gradient in coronary computed tomography angiography is a novel noninvasive approach to the identification of functionally significant coronary artery stenosis: a comparison with fractional flow reserve. J Am Coll Cardiol. 2013;61:12711279. doi: 10.1016/j.jacc.2012.12.029. 